EDMUND CHADA BARACAT ${ }^{1}$

JOSÉ MARIA SOARES-JUNIOR ${ }^{2}$

\title{
Ovários policísticos, resistência insulínica e síndrome metabólica
}

\section{Polycystic ovaries: Insulin resistance and metabolic syndrome}

A síndrome dos ovários policísticos (SOP) é a endocrinopatia mais comum na fase reprodutiva da mulher; com prevalência entre 4 e 12\%. A ocorrência familiar é freqüente, principalmente quando há parentesco de primeiro grau $(20 \text { a } 60 \%)^{1,2}$.

$\mathrm{O}$ conceito da SOP é muito amplo; caracteriza-se clinicamente pela presença de disfunção menstrual, hiperandrogenismo e anovulação crônica. Daí a importância de se normatizar alguns parâmetros para melhor definir essa síndrome. Em 1990, em reunião de consenso promovido pelo National Institute of Health decidiu-se incluir, entre as portadoras da síndrome, mulheres com hiperandrogenismo clínico ou laboratorial e ciclos espaniomenorréicos (menos que seis ciclos por ano), desde que afastadas outras alterações como síndrome de Cushing, hiperprolactinemia, deficiência enzimática da supra-renal e distúrbios da tireóide. Em 2003, o Consenso de Rotterdan, na Holanda, propôs como critérios diagnósticos:

- ciclos espaniomenorréicos ou amenorréia (anovulação crônica);

- sinais clínicos ou bioquímicos de hiperandrogenismo;

- presença de ovários policísticos, desde que fossem excluídas a deficiência enzimática da supra-renal, neoplasias de ovário secretora de androgênios e a síndrome de Cushing ${ }^{3}$.

Em 2006, a Sociedade para o Estudo de Excesso de Androgênios (Androgen Excess Society) estaleceram os seguintes critérios:

- hiperandrogenismo (hiursutismo e/ou hiperandrogenismo);

- disfunção ovariana (anovulação e/ou ovários policísticos);

- exclusão de outras endocrinopatias .

A etiologia da SOP ainda não está completamente elucidada; no entanto, várias hipóteses são aventadas, como alterações na liberação pulsátil de gonadotrofinas (GnRH); na liberação hipofisária dos hormônios luteinizante (LH) e folículo-estimulante (FSH); nas funções ovariana e supra-renal, e mais recentemente a resistência insulínica. Em revisão da literatura, Legro et al. (2004) $)^{5}$ relataram que aproximadamente 50 a $70 \%$ das mulheres com síndrome dos ovários policísticos (SOP) tinham resistência periférica à

Correspondência:

Edmund Chada Baracat e José Maria Soares Júnior Rua Sena Madureira, 1245, apto. 11

CEP 04021-051

Fone (11) 5576-4268 - Fax (11) 5576-4271

E-mail: jsoares415@hotmail.com

Recebido

20/03/2007

Aceito com modificações $13 / 04 / 2007$
I Professor Titular da Disciplina de Endocrinologia Ginecológica do Departamento de Ginecologia da Universidade Federal de São Paulo - UNIFESP - São Paulo (SP), Brasil; Professor Titular do Departamento de Obstetrícia e de Ginecologia da Universidade de São Paulo - USP - São Paulo (SP), Brasil.

2 Professor Adjunto da Disciplina de Endocrinologia Ginecológica do Departamento de Ginecologia da Universidade Federal de São Paulo - UNIFESP - São Paulo (SP), Brasil. 
insulina, que agravava o quadro de hiperandrogenismo. Entre os mecanismos envolvidos, salienta-se o estímulo direto pela insulina na síntese de androgênios nos ovários e nas supra-renais.

É importante lembrar que mulheres com SOP e resistência periférica à insulina podem apresentar-se com peso normal. Evidências demonstraram a ação da insulina e dos fatores insulinóides de crescimento (IGF I e II) sobre o desenvolvimento do folículo ovariano ${ }^{6-8}$ e sobre a estimulação da síntese de androgênios nas células da teca interna in vitro. Assim, a insulina poderia ter papel relevante na patogenia da afecção. Além disso, a hiperinsulinemia está relacionada com a redução da síntese de SHBG no fígado, bem como das proteínas carreadoras dos fatores insulinóides ${ }^{6}$.

A resistência insulínica caracteriza-se pela diminuição da sensibilidade dos tecidos à ação da insulina, gerando importantes implicações metabólicas. Nas mulheres com SOP, provocaria deterioração da função das células beta do pâncreas, levando a intolerância à glicose. Contudo, nem sempre é fácil identificar a paciente com resistência insulínica9 .

Dentre as diferentes técnicas descritas para análise da resistência insulínica, incluem-se:

- índice HOMA, medida da insulina em mUI/l x glicemia em mmol/dl / 22,5;

- teste de tolerância oral à glicose, que consiste na administração de $75 \mathrm{~g}$ de glicose e, a seguir, faz-se a determinação de glicemia e insulina nos tempos 0, 30, 60 e 120 minutos;

- teste de tolerância à glicose simplificado, no qual se faz as dosagens apenas nos tempos 0 e 120 minutos;

- relação entre glicemia e insulina (G/I) de jejum, cujo valor considerado normal é menor que 4,5;

- teste QUICKI (quantitative sensitivity check index), que consiste no resultado obtido com a seguinte fórmula matemática: QUICKI = $1 /$ [Log insulina de jejum insulin + Log glicemia de jejum $]^{9,10}$.

As mulheres obesas com SOP devem ser avaliadas quanto à presença de resistência à insulina. Deve-se ainda pesquisar a ocorrência de outros agravos como hipertensão arterial, dislipidemia, obesidade central e intolerância à glicose, como também o desenvolvimento da síndrome metabólica ${ }^{10}$.

As mudanças no estilo de vida observadas a partir da segunda metade do século XX, que incluíram alterações nos hábitos alimentares e a adoção de estilo de vida sedentário, contribuíram para o aumento da freqüência de dislipidemia, obesidade, diabete melito e hipertensão. A ocorrência concomitante de todas estas alterações, associada a um quadro de resistência insulínica, compõe a chamada síndrome metabólica, que cursa com importante aumento do risco de desenvolvimento de doenças cardiovasculares severas ${ }^{11}$.

A exata natureza dos mecanismos implicados nas alterações lipoprotéicas da síndrome de resistência insulínica não se encontra totalmente esclarecida, porém, a hiperinsulinemia tem papel central neste processo, visto que está implicada na modulação de enzimas-chave do metabolismo lipídico ${ }^{12}$. Os agonistas de receptores nucleares ativados pelos proliferadores do peroxisina (PPAR) são capazes de induzir a melhora na resistência insulínica e na dislipidemia associada ${ }^{12}$. Esse achado evidencia o papel relevante destes receptores na regulação de diversas vias metabólicas relacionadas com a homeostase glicídica, a pressão arterial, a adipogênese e o metabolismo lipídico.

As condições que cursam com hiperinsulinemia, em geral, estão associadas à chamada tríade lipídica: aumento moderado de triglicerídeos, redução do HDL-colesterol e presença de níveis aumentados de LDL. No adipócito, a resistência insulínica causa aumento na liberação de ácidos graxos livres, enquanto no fígado determina menor supressão na síntese de VLDL. O resultado desse processo é a liberação de um excesso de partículas de VLDL grandes, ricas em triglicerídeos, que, por sua vez, geram uma cascata de eventos de troca que culminam com a redução nos níveis de HDL. Outras alterações, tais como a redução da ação da lipoproteíno-lipase e o aumento da ação da lipase hepática, são também necessárias para a completa expressão fenotípica da tríade lipídica, contribuindo para a transformação de LDL em partículas de menor diâmetro e maior densidade, e ainda para a manutenção de um estado de lipemia pós-prandial, com a circulação de lipoproteínas remanescentes ricas em colesterol ${ }^{10}$.

O conceito de síndrome metabólica engloba, pois, um estado de anormalidades clínicas e laboratoriais associado a maior risco de desenvolvimento de doenças cardiovasculares. O perfil lipídico clássico dessa síndrome se caracteriza por elevação dos triglicerídeos e elevação dos níveis de LDL, bem como redução do HDL-colesterol. Essas condições somam-se aos demais componentes para determinar elevação do risco cardiovascular e morte prematura, que podem ocorrer nas mulheres com síndrome de ovários policísticos, obesidade, resistência insulínica e síndrome metabólica ${ }^{13}$.

Por estas razões, o adequado acompanhamento e aconselhamento das pacientes com SOP é fundamental para evitar as complicações e os eventuais riscos relacionados a essa síndrome. 


\section{Referências}

1. Solomon CG. The epidemiology of polycystic ovary syndrome. Prevalence and associated disease risks. Endocrinol Metab Clin North Am. $1999 ; 28(2): 247-63$.

2. Legro RS, Barnhart HX, Schlaff WD, Carr BR, Diamond MP, Carson SA, Steinkampf MP, Coutifaris C, McGovern PG, Cataldo NA, Gosman GG, Nestler JE, Giudice LC, Leppert PC, Myers ER; Cooperative Multicenter Reproductive Medicine Network. Clomiphene, metformin, or both for infertility in the polycystic ovary syndrome. N Engl J Med. 2007;356(6):551-66.

3. Trivax B, Azziz R. Diagnosis of polycystic ovary syndrome. Clin Obstet Gynecol. 2007;50(1):168-77.

4. Azziz R, Carmina E, Dewailly D, Diamanti-Kandarakis E, Escobar-Morreale HF, Futterweit W, Janssen OE, Legro RS, Norman R, Taylor AE, Witchel SF; Androgen Excess Society. Positions statement: criteria for defining polycystic ovary syndrome as apredominantly hyperandrogenic syndrome: an Androgen Excess Society guideline. J Clin Endocrinol Metab. 2006;91(1 1):4237-45.

5. Maciel GA, Soares Junior JM, Alves da Motta EL, Abi Haidar M, de Lima GR, Baracat EC. Nonobese women with polycystic ovary syndrome respond better than obese women to treatment with metformin. Fertil Steril. 2004;81 (2):355-60.

6. Teixeira Filho FL, Baracat EC, Lee TH, Suh CS, Matsui M, Chang RJ, Shimasaki S, Erickson GF. Aberrant expression of growth differentiation factor-9 in oocytes of women with polycystic ovary syndrome. J Clin Endocrinol Metab. 2002;87(3):1337-44.

7. Legro RS, Castracane VD, Kauffman RP. Detecting insulin resistance in polycystic ovary syndrome: purposes and piffalls. Obstet Gynecol Surv. 2004;59(2):141-54. Review.

8. Giudice LC. Endometrium in PCOS: Implantation and predisposition to endocrine CA. Best Pract Res Clin Endocrinol Metab. 2006;20(2):235-44.

9. Barber TM, Wass JA, McCarthy MI, Franks S. Metabolic characteristics of women with polycystic ovaries and oligo-amenorrhoea but normal androgen levels: implications for the management of polycystic ovary syndrome. Clin Endocrinol (Oxf). 2007;66(4):513-7.

10. de Paula Martins W, Santana LF, Nastri CO, Ferriani FA, de Sa MF, Dos Reis RM. Agreement among insulin sensitivity indexes on the diagnosis of insulin resistance in polycystic ovary syndrome and ovulatory women. Eur J Obstet Gynecol Reprod Biol. 2007 Jan 4; [Epub ahead of print]

11. Burke GL, Bell RA. Trends in cardiovascular disease: incidence and risk factors. In: Wong ND, Black HR, Gardin JM, editors.. Preventive cardiology. New York: Mc-Graw-Hill; 2000. p.21-46.

12. Verges B. Clinical interest of PPARs ligands. Diabetes Metab. 2004;30(1):7-12.

13. Stolar M. Metabolic syndrome: controversial but useful. Cleve Clin J Med. 2007;74(3):199-202, 205-8. 